\title{
PERFORMANCE INVESTIGATION OF CLOSED LOOP PULSATING HEAT PIPE WITH ACETONE AS WORKING FLUID
}

\author{
Roshan D.Bhagat ${ }^{1}$, K.M.Watt ${ }^{2}$ \\ ${ }^{1}$ Student M.E.Thermal Engineering, Prof. Ram Meghe Institute of Technology \& Research Badnera-Amravati, Sant \\ Gadge Baba Amravati University \\ ${ }^{2}$ Department of Mechanical Engineering, Prof. Ram Meghe Institute of Technology \& Research Badnera-Amravati, \\ Sant Gadge Baba Amravati University
}

\begin{abstract}
The closed-loop pulsating heat pipe is a type of small heat transfer device with a very high thermal conductivity. It was invented to meet the requirement for smaller heat transfer devices. The objective of this work is to study thermal performance of closed loop pulsating heat pipe with acetone as working fluid. Copper has been selected as material for heat pipe due to compatibility of copper with acetone as working fluid. Filling ratio of the working fluid significantly influence on the performance closed loop pulsating heat pipe. From the past studies it was observed that filling ratio of 30-75\% provides the best result hence $60 \%$ filling ratio has been selected for this filing ratio the thermal performance of closed loop pulsating heat pipe with acetone as working fluid is investigated.
\end{abstract}

Keywords: closed loop pulsating heat pipe, condenser, evaporator, working fluid, filling ratio.

\section{INTRODUCTION}

The closed-loop pulsating heat pipe is a type of small heat transfer device with a very high thermal conductivity. It was invented to meet the requirement for smaller heat transfer devices. It can transfer sufficient heat for heat dissipation applications in modern electronic devices. The Closed loop pulsating heat pipe is made of a long copper capillary tube, bent into an undulating tube and connected at the ends to form a closed-loop with no internal wick structure [1]. Working fluid is partially filled in the tube. The closed loop pulsating heat pipe has a condenser, evaporator section and adiabatic section. As any other two-phase passive thermal control device, heat is acquired from the source through the evaporator section transferring it to the working fluid where the slug/plug pumping action will be generated. The fluid then flows by the adiabatic section towards the condenser section. On a closed loop configuration, the fluid is allowed to circulate and after being condensed, the fluid returns to the evaporator section to complete the loop. The tube is evacuated and consequently partially filled with working fluid. Since an inner diameter of the tube is very small and then meets a capillary scale, the inside working fluid forms into liquid slugs alternating with vapour plugs along the entire length of the tube [2].

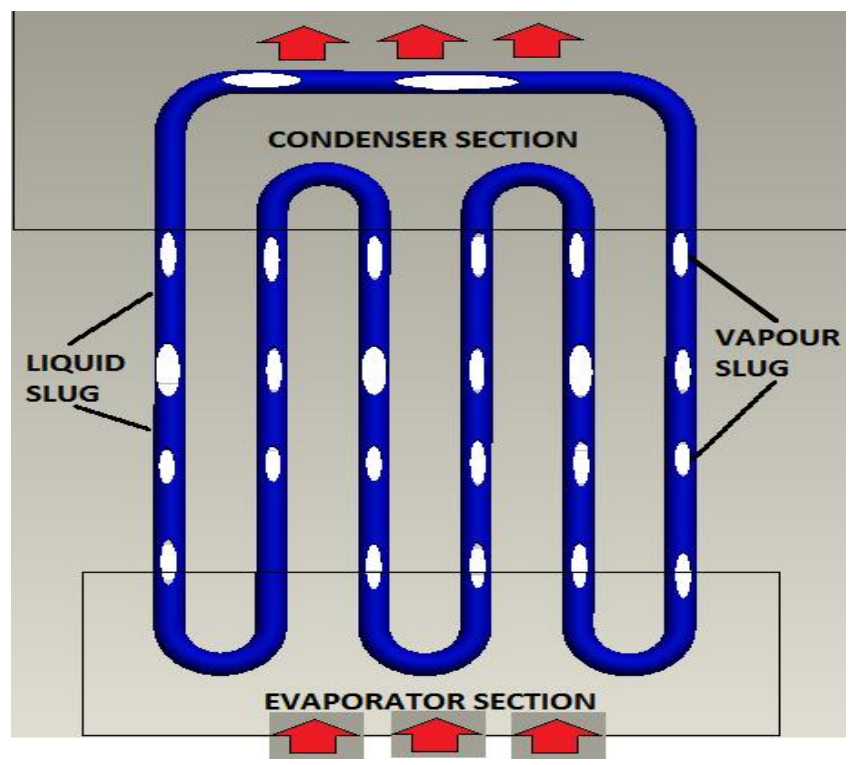

Fig 1: Closed loop pulsating heat pipe

When one end of the closed-loop pulsating heat pipe, called 'evaporator section', is subjected to heat or high temperature, the working fluid, which is in liquid slug form, will evaporate, expand, and move through the no heat transferring zone, or 'adiabatic section', toward a cooler Section, 'condenser section'. Then, the vapour plugs will condense, collapse, and release the heat into the environment. Therefore, the vapour plug evaporating in the evaporator section will consequently flow to replace the vapour plug collapsing in the condenser section. Due to this mechanism, the working fluid can circulate and continuously transfer heat in a cycle. The structure of the closed loop pulsating heat pipe is as shown in Figure 1. 
Table 1: Compatibility of closed loop pulsating heat pipe material with the working fluid [2]

\begin{tabular}{|l|l|}
\hline Working fluid & Compatible Material \\
\hline Methanol & $\begin{array}{l}\text { Stainless Steel, Iron, Copper, Brass, } \\
\text { Silica, Nickel }\end{array}$ \\
\hline Acetone & Stainless Steel, Copper, Brass, Silica \\
\hline
\end{tabular}

Table 2: Boiling point and operating ranges of working fluid [2]

\begin{tabular}{|l|l|l|}
\hline Working fluid & $\begin{array}{l}\text { Boiling point At 1 } \\
\text { atm in K }\end{array}$ & $\begin{array}{l}\text { Temperature } \\
\text { ranges in K }\end{array}$ \\
\hline Acetone & 329.4 & $273-393$ \\
\hline Methanol & 337.8 & $283-403$ \\
\hline Ethanol & 351.5 & $273-403$ \\
\hline
\end{tabular}

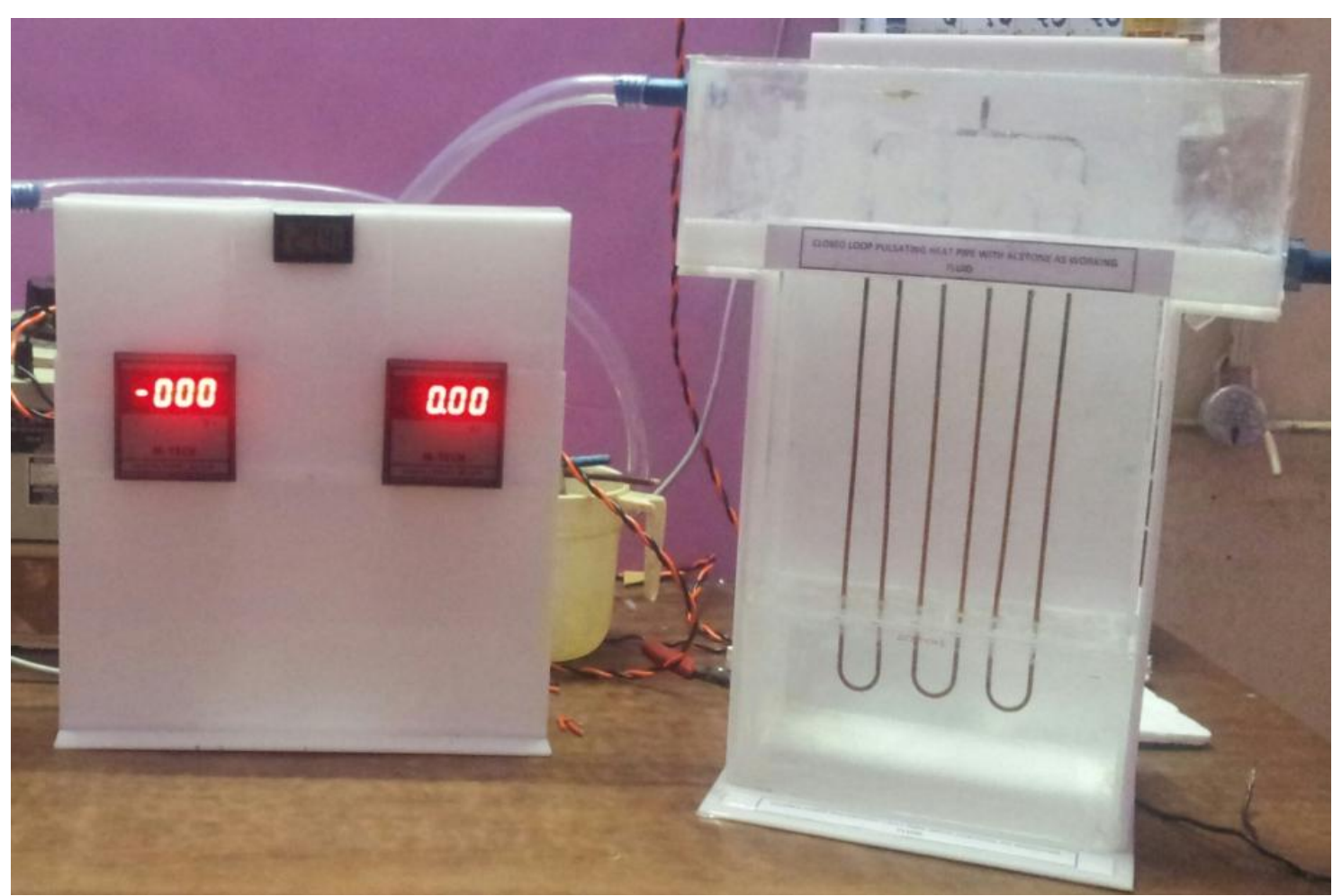

Fig 2: Experimental setup of closed loop pulsating heat pipe with acetone as working fluid

\section{EXPERIMENTATION AND TESTING CLOSED LOOP PULSATING HEAT PIPE WITH ACETONE AS WORKING FLUID}

Table 3: Evaporator temperature of closed loop pulsating heat pipe with acetone as working fluid at variable heat input

\begin{tabular}{|c|c|c|c|c|c|c|c|c|c|c|}
\hline \multirow[t]{2}{*}{ S.N } & \multirow[t]{2}{*}{$\begin{array}{l}\text { VOLTAGE } \\
(\mathrm{V})\end{array}$} & \multirow[t]{2}{*}{$\begin{array}{l}\text { CURRENT } \\
\text { (A) }\end{array}$} & \multirow{2}{*}{$\begin{array}{l}\text { HEAT } \\
\text { INPUT } \\
\text { (Watt) }\end{array}$} & \multicolumn{6}{|c|}{$\begin{array}{l}\text { EVAPORATOR TEMPERATURE } \\
\text { OF ACETONE } I N^{0} C\end{array}$} & \multirow{2}{*}{$\begin{array}{l}\text { AVERAGE } \\
\text { EVAPORATOR } \\
\text { TEMPERATURE }\end{array}$} \\
\hline & & & & $\mathrm{T}_{1}$ & $\mathrm{~T}_{2}$ & $\mathrm{~T}_{3}$ & $\mathrm{~T}_{4}$ & $\mathrm{~T}_{5}$ & $\mathrm{~T}_{6}$ & \\
\hline 1 & 10 & 0.1 & 1 & 26 & 26.1 & 25.8 & 26 & 26.1 & 25.9 & 25.98333333 \\
\hline 2 & 20 & 0.2 & 4 & 26 & 26.1 & 25.9 & 25.7 & 25.5 & 25.8 & 25.83333333 \\
\hline 3 & 50 & 0.53 & 26.5 & 29 & 29 & 29 & 29.1 & 29.1 & 29.2 & 29.06666667 \\
\hline 4 & 60 & 0.63 & 37.8 & 30 & 30.1 & 30.2 & 30.2 & 30.1 & 30.1 & 30.11666667 \\
\hline 5 & 70 & 0.73 & 51.1 & 31.8 & 31.7 & 31.7 & 31.6 & 31.7 & 31.7 & 31.7 \\
\hline 6 & 80 & 0.84 & 67.2 & 34 & 33.9 & 33.9 & 33.9 & 33.9 & 33.8 & 33.9 \\
\hline 7 & 90 & 0.94 & 84.6 & 36.9 & 36.9 & 36.9 & 36.6 & 36.7 & 36.6 & 36.76666667 \\
\hline 8 & 100 & 1.04 & 104 & 39.9 & 40.2 & 40.2 & 40.2 & 40.2 & 40.2 & 40.15 \\
\hline 9 & 165 & 1.73 & 285.45 & 65.1 & 65.1 & 65.1 & 65.1 & 65.1 & 65.1 & 65.1 \\
\hline 10 & 175 & 1.77 & 309.75 & 66.5 & 66.5 & 66.8 & 67.1 & 67.1 & 67.1 & 66.85 \\
\hline 11 & 185 & 1.95 & 360.75 & 68.7 & 68.7 & 68.7 & 68.5 & 68.5 & 68.8 & 68.65 \\
\hline
\end{tabular}


Table 4: Condenser temperature of closed loop pulsating heat pipe with acetone as working fluid at variable heat input

\begin{tabular}{|c|c|c|c|c|c|c|c|c|c|c|}
\hline \multirow[t]{2}{*}{ S.N } & \multirow[t]{2}{*}{$\begin{array}{l}\text { VOLTAGE } \\
\text { (V) }\end{array}$} & \multirow[t]{2}{*}{$\begin{array}{l}\text { CURRENT } \\
\text { (A) }\end{array}$} & \multirow{2}{*}{$\begin{array}{l}\text { HEAT } \\
\text { INPUT } \\
\text { (Watt) }\end{array}$} & \multicolumn{6}{|c|}{$\begin{array}{l}\text { CONDENSER TEMPERATURE } \\
\text { OF ACETONE } I N^{0} C\end{array}$} & \multirow{2}{*}{$\begin{array}{l}\text { AVERAGE } \\
\text { CONDENSER } \\
\text { TEMPERATURE }\end{array}$} \\
\hline & & & & $T_{7}$ & $T_{8}$ & $T_{9}$ & $T_{10}$ & $T_{11}$ & $T_{12}$ & \\
\hline 1 & 10 & 0.1 & 1 & 25.8 & 26 & 25.8 & 26.1 & 25.8 & 26.1 & 25.93333333 \\
\hline 2 & 20 & 0.2 & 4 & 25.8 & 25.9 & 25.8 & 25.8 & 25.7 & 25.9 & 25.81666667 \\
\hline 3 & 50 & 0.53 & 26.5 & 25.5 & 25.5 & 25.5 & 25.5 & 25.3 & 25.5 & 25.46666667 \\
\hline 4 & 60 & 0.63 & 37.8 & 25 & 25.1 & 25 & 25 & 25 & 25 & 25.01666667 \\
\hline 5 & 70 & 0.73 & 51.1 & 25.2 & 25.1 & 25 & 24.9 & 25 & 25.2 & 25.06666667 \\
\hline 6 & 80 & 0.84 & 67.2 & 25.5 & 25.5 & 25.5 & 25.5 & 25.2 & 25.3 & 25.41666667 \\
\hline 7 & 90 & 0.94 & 84.6 & 26.4 & 26.4 & 26.4 & 26.1 & 25.9 & 25.7 & 26.15 \\
\hline 8 & 100 & 1.04 & 104 & 26.9 & 27 & 27.2 & 27.3 & 27.3 & 27 & 27.11666667 \\
\hline 9 & 165 & 1.73 & 285.45 & 31.7 & 32.6 & 32.9 & 32.7 & 31.7 & 30.6 & 32.03333333 \\
\hline 10 & 175 & 1.77 & 309.75 & 32.4 & 32.3 & 32.5 & 32.5 & 32 & 30.7 & 32.06666667 \\
\hline 11 & 185 & 1.95 & 360.75 & 33 & 33.2 & 33.2 & 32.8 & 32.8 & 30.7 & 32.61666667 \\
\hline
\end{tabular}

Table 5: Thermal resistance of closed loop pulsating heat pipe with acetone as working fluid at variable heat input

\begin{tabular}{|l|l|l|}
\hline S.N & $\begin{array}{l}\text { HEAT INPUT (Watt) } \\
\text { FOR ACETONE }\end{array}$ & $\begin{array}{l}\text { THERMAL RESISTANCE } \\
\text { OF ACETONE }\end{array}$ \\
\hline 1 & 1 & 0.05 \\
\hline 2 & 4 & 0.004166667 \\
\hline 3 & 26.5 & 0.135849057 \\
\hline 4 & 37.8 & 0.134920635 \\
\hline 5 & 51.1 & 0.129810828 \\
\hline 6 & 67.2 & 0.126240079 \\
\hline 7 & 84.6 & 0.125492514 \\
\hline 8 & 104 & 0.125320513 \\
\hline 9 & 285.45 & 0.115840486 \\
\hline 10 & 309.75 & 0.112294861 \\
\hline 11 & 360.75 & 0.0998845 \\
\hline
\end{tabular}

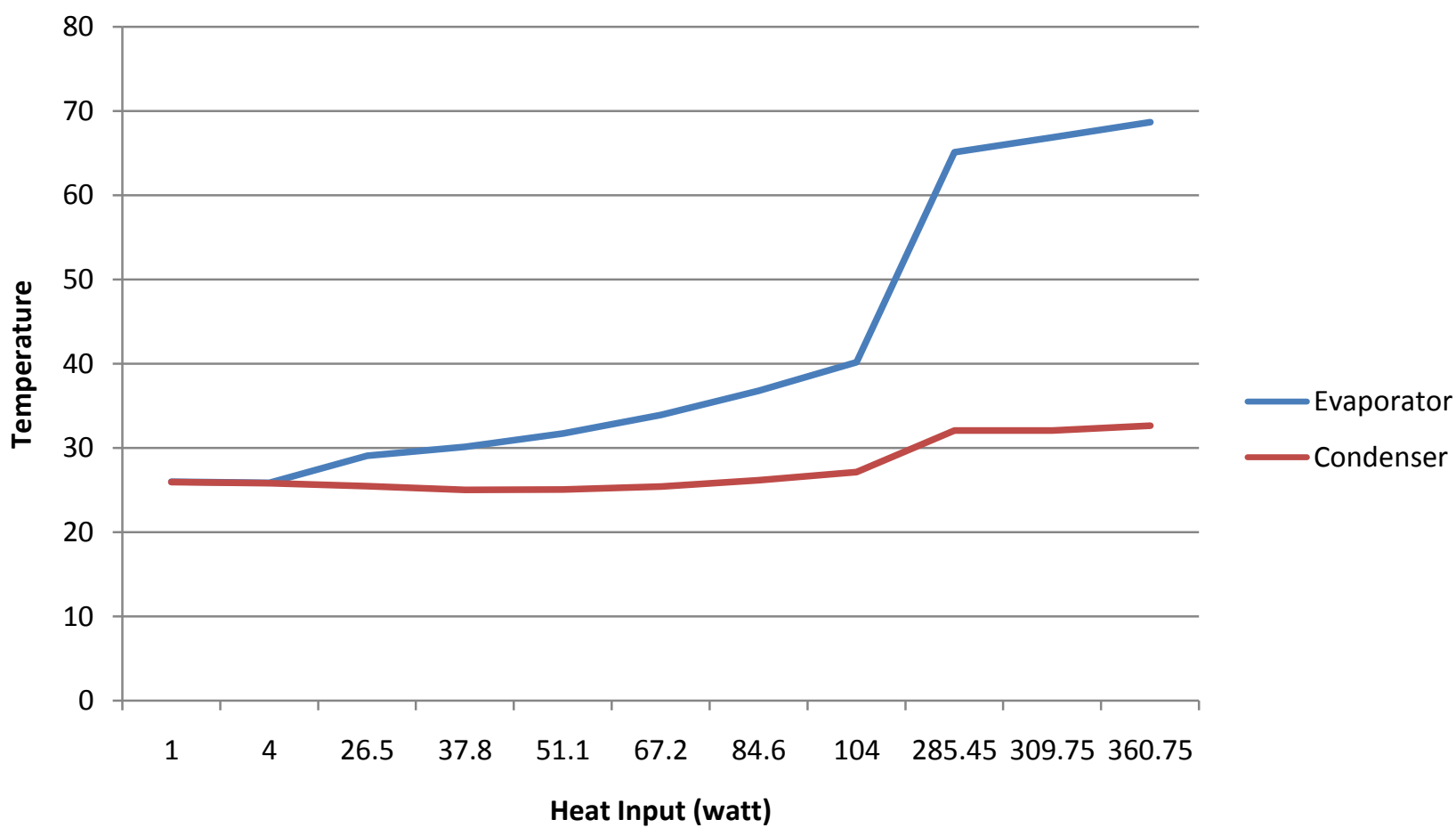

Fig 3: Evaporator and condenser temperature of closed loop pulsating heat pipe with acetone as working fluid at variable heat input 


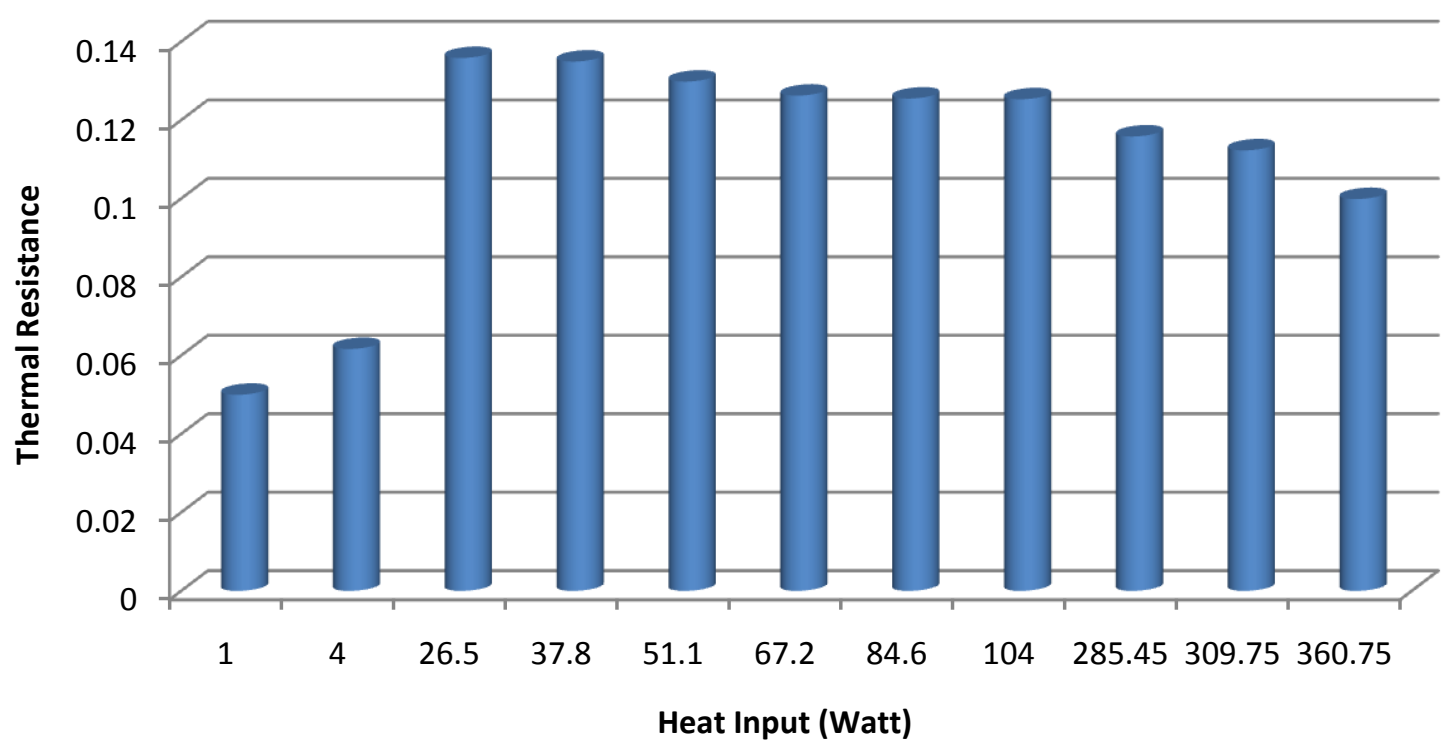

Fig 4: Thermal resistance of closed loop pulsating heat pipe with acetone as working fluid at variable heat input

$$
\begin{gathered}
R_{\text {thermal }}=\frac{T_{e}-T_{c}}{Q} \\
R_{\text {thermal }}-\text { Thermal resistance } \\
T_{e}-\text { average evaporator temperature } \\
T_{c}-\text { average condenser temperature }
\end{gathered}
$$

\section{CONCLUSION}

The thermal resistance of closed loop pulsating heat pipe with acetone as working fluid decreases with increase in the heat input hence the thermal performance of closed loop pulsating heat pipe with acetone as working fluid increases with increase in the heat input. Also it was found from the experimental results that thermal resistance of closed loop pulsating heat pipe with acetone as working fluid increases first and then decreases.

\section{REFERENCES}

[1] H. Akachi, F. Polasek, and P. Stulc, "Pulsating heat pipes," in Proc. 5th Intl. Heat Pipe Symp., Melbourne, Australia, 1996, pp. 208-217.

[2] M.B. Shafii, A. Faghri, Y. Zhang, Thermal modeling of unlooped and looped pulsating heat pipes, ASME J. Heat Transfer 123 (2001) 1159-1172.

[3] T. N. Wong, "High speed flow visualization of a closed loop pulsating heat pipe," Heat Mass Transfer, vol. 48, pp. 3338-3351, 2005.

[4] N. Soponpongpipat, P. Sakulchangsatjatai, N. Kammuang-lue, and P. Terdtoon, "Investigation of the start up condition of a closed loop oscillating heat pipe," Heat Transfer Eng., vol. 30, no. 8, pp. 626642, 2009.

[5] Khandekar, S., Dollinger, N., Groll, M., "Understanding Operational Regimes of Closed Loop Pulsating Heat Pipes: An Experimental Study", 2003, Applied Thermal Engineering, Vol. 23, pp.707-719.
[6] T. Mallikharjuna Rao, Dr. S. S. Rao, Heat Pipes for Steam Condensation, IOSR Journal of Mechanical and Civil Engineering (IOSR-JMCE) e-ISSN: 22781684, ISSN: 2320-334X, Volume 11, Issue 2 Ver. I (Mar- Apr. 2014), PP 16-19.

[7] M. Groll, S. Khandekar, Pulsating heat pipes, Proceedings of the 3rd International Conference on Transport Phenomena in Multiphase Systems, Kielce, Poland, 2002, 35-44 (ISBN83-88906-03-08)

[8] S. Khandekar, M. Schneider, R. Kulenovic, M. Groll, Thermofluid dynamic study of flat plate closed loop pulsating heat pipes, Microsc. Thermophys. Eng. 6 (4) (2002) 303-318 (ISSN 1089-3954)

[9] Niti Kammuang-lue, Kritsada, Phrut Sakulchangsatjatai, Pradit Terdtoon, Correlation to Predict Thermal Performance According to Working Fluids of Vertical Closed-Loop Pulsating Heat Pipe, International Journal of Mechanical, Aerospace, Industrial and Mechatronics Engineering Vol:8 No:5, 2014

[10] Zhang, Y., Faghri, A., "Heat Transfer in a Pulsating Heat pipe with an Open End", International Journal of Heat and Mass Transfer, Vol. 45, 2002, pp. 755764. 